\title{
Levenberg-Marquardt Dynamics Associated to Variational Inequalities
}

\author{
Radu Ioan Boț ${ }^{1} \cdot$ Ernö Robert Csetnek ${ }^{1}$
}

Received: 13 June 2016 / Accepted: 10 April 2017 / Published online: 18 May 2017

(C) The Author(s) 2017. This article is an open access publication

Abstract In connection with the optimization problem

$$
\inf _{x \in \operatorname{argmin} \Psi}\{\Phi(x)+\Theta(x)\},
$$

where $\Phi$ is a proper, convex and lower semicontinuous function and $\Theta$ and $\Psi$ are convex and smooth functions defined on a real Hilbert space, we investigate the asymptotic behavior of the trajectories of the nonautonomous Levenberg-Marquardt dynamical system

$$
\left\{\begin{array}{l}
v(t) \in \partial \Phi(x(t)) \\
\lambda(t) \dot{x}(t)+\dot{v}(t)+v(t)+\nabla \Theta(x(t))+\beta(t) \nabla \Psi(x(t))=0,
\end{array}\right.
$$

where $\lambda$ and $\beta$ are functions of time controlling the velocity and the penalty term, respectively. We show weak convergence of the generated trajectory to an optimal solution as well as convergence of the objective function values along the trajectories, provided $\lambda$ is monotonically decreasing, $\beta$ satisfies a growth condition and a relation expressed via the Fenchel conjugate of $\Psi$ is fulfilled. When the objective function is assumed to be strongly convex, we can even show strong convergence of the trajectories.

Keywords Nonautonomous systems $\cdot$ Levenberg-Marquardt dynamics $\cdot$ Regularized Newton-like dynamics · Lyapunov analysis · Convex optimization · Variational inequalities $\cdot$ Penalization techniques

Mathematics Subject Classification (2010) $34 \mathrm{G} 25 \cdot$ 47J25 · 47H05 · 90C25

Research of the second author supported by FWF (Austrian Science Fund), Lise Meitner Programme, project M 1682-N25.

Radu Ioan Boţ

radu.bot@univie.ac.at.

Ernö Robert Csetnek

ernoe.robert.csetnek@univie.ac.at

1 Faculty of Mathematics, University of Vienna, Oskar-Morgenstern-Platz 1, 1090 Vienna, Austria 


\section{Introduction}

Throughout this manuscript $\mathcal{H}$ is assumed to be a real Hilbert space endowed with inner product $\langle\cdot, \cdot\rangle$ and associated norm $\|\cdot\|=\sqrt{\langle\cdot, \cdot\rangle}$. When $T: \mathcal{H} \rightarrow \mathcal{H}$ is a $C^{1}$ operator with derivative $T^{\prime}$, the solving of the equation

$$
\text { find } x \in \mathcal{H} \text { such that } T x=0
$$

can be approached by the classical Newton method, which generates an approximating sequence $\left(x_{n}\right)_{n \geq 0}$ of a solution of the operator equation through

$$
T\left(x_{n}\right)+T^{\prime}\left(x_{n}\right)\left(x_{n+1}-x_{n}\right)=0 \forall n \geq 0 .
$$

In order to overcome the fact that the classical Newton method assumes the solving of an equation which is in general not well-posed, one can use instead the Levenberg-Marquardt method

$$
T\left(x_{n}\right)+\left(\lambda_{n} \operatorname{Id}+T^{\prime}\left(x_{n}\right)\right)\left(\frac{x_{n+1}-x_{n}}{\Delta t_{n}}\right)=0 \forall n \geq 0,
$$

where Id $: \mathcal{H} \rightarrow \mathcal{H}$ denotes the identity operator on $\mathcal{H}, \lambda_{n}$ a regularizing parameter and $\Delta t_{n}>0$ the step size.

When $T: \mathcal{H} \rightrightarrows \mathcal{H}$ is a (set-valued) maximally monotone operator, Attouch and Svaiter showed in [10] that the above Levenberg-Marquardt algorithm can be seen as a time discretization of the dynamical system

$$
\left\{\begin{array}{l}
v(t) \in T(x(t)) \\
\lambda(t) \dot{x}(t)+\dot{v}(t)+v(t)=0
\end{array}\right.
$$

for approaching the inclusion problem

$$
\text { find } x \in \mathcal{H} \text { such that } 0 \in T x \text {. }
$$

This includes as a special instance the problem of minimizing a proper, convex and lower semicontinuous function, when $T$ is taken as its convex subdifferential. Later on, this investigation has been continued in [2] in the context of minimizing the sum of a proper, convex and lower semicontinuous function with a convex and smooth one.

In the spirit of [10], we approach in this paper the optimization problem

$$
\inf _{x \in \operatorname{argmin} \Psi}\{\Phi(x)+\Theta(x)\},
$$

where $\Phi: \mathcal{H} \rightarrow \mathbb{R} \cup\{+\infty\}$ is a proper, convex and lower semicontinuous function and $\Psi, \Theta: \mathcal{H} \rightarrow \mathbb{R}$ are convex and smooth functions, via the Levenberg-Marquardt dynamical system

$$
\left\{\begin{array}{l}
v(t) \in \partial \Phi(x(t)) \\
\lambda(t) \dot{x}(t)+\dot{v}(t)+v(t)+\nabla \Theta(x(t))+\beta(t) \nabla \Psi(x(t))=0,
\end{array}\right.
$$

where $\lambda$ and $\beta$ are functions of time controlling the velocity and the penalty term, respectively.

If $\partial \Phi+N_{\operatorname{argmin}} \Psi$ is maximally monotone, then determining an optimal solution $x \in \mathcal{H}$ of (3) means nothing else than solving the subdifferential inclusion problem

$$
\text { find } x \in \mathcal{H} \text { such that } 0 \in \partial \Phi(x)+\nabla \Theta(x)+N_{\operatorname{argmin}} \Psi(x)
$$

or, equivalently, solving the variational inequality

find $x \in \operatorname{argmin} \Psi$ and $v \in \partial \Phi(x)$ such that $\langle v+\nabla \Theta(x), y-x\rangle \geq 0 \forall y \in \operatorname{argmin} \Psi$. (6) 
We show weak convergence of the trajectory $x(\cdot)$ generated by (4) to an optimal solution of (3) as well as convergence of the objective function values along the trajectory to the optimal objective value, provided the assumption

$$
\forall p \in \operatorname{ran} N_{\operatorname{argmin}} \Psi \int_{0}^{+\infty} \beta(t)\left[\Psi^{*}\left(\frac{p}{\beta(t)}\right)-\sigma_{\operatorname{argmin}} \Psi\left(\frac{p}{\beta(t)}\right)\right] d t<+\infty
$$

is fulfilled and the functions $\lambda, \beta$ satisfy some mild conditions. If the objective function of (3) is strongly convex, the trajectory $x(\cdot)$ converges even strongly to the unique optimal solution of (3).

The condition (7) has its origins in the paper of Attouch and Czarnecki [5], where the solving of

$$
\inf _{x \in \operatorname{argmin} \Psi} \Phi(x)
$$

for $\Phi, \Psi: \mathcal{H} \rightarrow \mathbb{R} \cup\{+\infty\}$ proper, convex and lower semicontinuous functions, is approached through the nonautonomous first order dynamical system

$$
0 \in \dot{x}(t)+\partial \Phi(x(t))+\beta(t) \partial \Psi(x(t)),
$$

by assuming that the penalizing function $\beta:[0,+\infty) \rightarrow(0,+\infty)$ tend to $+\infty$ as $t \rightarrow$ $+\infty$. Several ergodic and nonergodic convergence results have been reported in [5] under the key assumption (7).

The paper of Attouch and Czarnecki [5] was the starting point of a remarkable number of research articles devoted to penalization techniques for solving optimization problems of type (3), but also generalizations of the latter in form of variational inequalities expressed with maximal monotone operators (see [3, 5-9, 12, 14-17, 20, 21]). In the literature enumerated above, the monotone inclusions problems have been approached either through continuous dynamical systems or through their discrete counterparts formulated as splitting algorithms. We speak in both cases about methods of penalty type, which means in this context that the operator describing the underlying set of the variational inequality under investigation is evaluated as a penalty functional. In the above-listed references one can find more general formulations of the key assumption (7), but also further examples for which these conditions are satisfied. In Remark 5 and Remark 6 we provide more insights into the relations of the dynamical system (14) to other continuous systems (and their discrete counterparts) from the literature.

The results we obtain in this paper are in the spirit of Attouch-Czarnecki [5]. However, since the dynamical system we focus on is a combination of two different types of dynamical systems, the asymptotic analysis is more involved, in the sense that one has to take into consideration the particularities of both continuous systems.

\section{Preliminaries}

In this section we present some preliminary definitions, results and tools that will be useful throughout the paper. We consider the following definition of an absolutely continuous function.

Definition 1 (see, for instance, [2,10]) A function $x:[0, b] \rightarrow \mathcal{H}$ (where $b>0$ ) is said to be absolutely continuous if one of the following equivalent properties holds: 
(i) there exists an integrable function $y:[0, b] \rightarrow \mathcal{H}$ such that

$$
x(t)=x(0)+\int_{0}^{t} y(s) d s \quad \forall t \in[0, b] ;
$$

(ii) $x$ is continuous and its distributional derivative is Lebesgue integrable on $[0, b]$;

(iii) for every $\varepsilon>0$, there exists $\eta>0$ such that for any finite family of intervals $I_{k}=$ $\left(a_{k}, b_{k}\right) \subseteq[0, b]$ we have the implication

$$
\left(I_{k} \cap I_{j}=\emptyset \text { and } \sum_{k}\left|b_{k}-a_{k}\right|<\eta\right) \Longrightarrow \sum_{k}\left\|x\left(b_{k}\right)-x\left(a_{k}\right)\right\|<\varepsilon .
$$

A function $x:[0,+\infty) \rightarrow \mathcal{H}$ (where $b>0$ ) is said to be locally absolutely continuous if it is absolutely continuous on each interval $[0, b]$ for $0<b<+\infty$.

Remark 1 (a) It follows from the definition that an absolutely continuous function is differentiable almost everywhere, its derivative coincides with its distributional derivative almost everywhere and one can recover the function from its derivative $\dot{x}=y$ by the integration formula (i).

(b) If $x:[0, b] \rightarrow \mathcal{H}$, where $b>0$, is absolutely continuous and $B: \mathcal{H} \rightarrow \mathcal{H}$ is $L$ Lipschitz continuous for $L \geq 0$, then the function $z=B \circ x$ is absolutely continuous, too. This can be easily seen by using the characterization of absolute continuity in Definition 1(iii). Moreover, $z$ is differentiable almost everywhere on $[0, b]$ and the inequality $\|\dot{z}(t)\| \leq L\|\dot{x}(t)\|$ holds for almost every $t \in[0, b]$.

The following results, which can be interpreted as continuous counterparts of the quasiFejér monotonicity for sequences, will play an important role in the asymptotic analysis of the trajectories of the dynamical system investigated in this paper. For the proof of Lemma 2 we refer the reader to [2, Lemma 5.1]. Lemma 3 follows by using similar arguments as used in [2, Lemma 5.2].

Lemma 2 Suppose that $F:[0,+\infty) \rightarrow \mathbb{R}$ is locally absolutely continuous and bounded from below and that there exists $G \in L^{1}([0,+\infty))$ such that for almost every $t \in[0,+\infty)$

$$
\frac{d}{d t} F(t) \leq G(t) .
$$

Then there exists $\lim _{t \rightarrow+\infty} F(t) \in \mathbb{R}$.

Lemma 3 If $1 \leq p<\infty, 1 \leq r \leq \infty, F:[0,+\infty) \rightarrow[0,+\infty)$ is locally absolutely continuous, $F \in L^{p}([0,+\infty)) \cap L^{\infty}([0,+\infty)), G_{1}, G_{2}:[0,+\infty) \rightarrow \mathbb{R}, G_{1} \in L^{1}([0,+\infty))$, $G_{2} \in L^{r}([0,+\infty))$ and for almost every $t \in[0,+\infty)$

$$
\frac{d}{d t} F(t) \leq G_{1}(t)+G_{2}(t)
$$

then $\lim _{t \rightarrow+\infty} F(t)=0$.

Proof In case $r=1$ this follows from Lemma 2 and the fact that $F \in L^{p}([0,+\infty))$.

Assume now that $r>1$ and define $q:=1+p\left(1-\frac{1}{r}\right)>1$, which fulfills the relation

$$
\frac{q-1}{p}+\frac{1}{r}=1 \text {. }
$$


Further, from (10) we derive for almost every $t \in[0,+\infty)$

$$
\frac{d}{d t}(F(t))^{q} \leq q(F(t))^{q-1} G_{1}(t)+q(F(t))^{q-1} G_{2}(t) .
$$

Since $F \in L^{\infty}([0,+\infty))$ and $G_{1} \in L^{1}([0,+\infty))$, the function $t \rightarrow(F(t))^{q-1} G_{1}(t)$ is $L^{1}$ integrable on $[0,+\infty)$. Moreover, due to $F^{q-1} \in L^{\frac{p}{q-1}}([0,+\infty)), G_{2} \in L^{r}([0,+\infty))$ and (11), the function $t \rightarrow(F(t))^{q-1} G_{2}(t)$ is also $L^{1}$-integrable on $[0,+\infty)$. We conclude that the function on the right-hand side of inequality (12) belongs to $L^{1}([0,+\infty))$. Applying now Lemma 2 we obtain that there exists $\lim _{t \rightarrow+\infty} F(t) \in \mathbb{R}$, which combined again with $F \in L^{p}([0,+\infty))$ delivers the conclusion.

The next result which we recall here is the continuous version of the Opial Lemma.

Lemma 4 Let $S \subseteq \mathcal{H}$ be a nonempty set and $x:[0,+\infty) \rightarrow \mathcal{H}$ a given function. Assume that

(i) for every $x^{*} \in S, \lim _{t \rightarrow+\infty}\left\|x(t)-x^{*}\right\|$ exists;

(ii) every weak sequential cluster point of the map $x$ belongs to $S$.

Then there exists $x_{\infty} \in S$ such that $x(t)$ converges weakly to $x_{\infty}$ as $t \rightarrow+\infty$.

\section{A Levenberg-Marquardt Dynamical System: Existence and Uniqueness of the Trajectories}

Consider the optimization problem

$$
\inf _{x \in \operatorname{argmin} \Psi}\{\Phi(x)+\Theta(x)\},
$$

where $\mathcal{H}$ is a real Hilbert space and the following conditions hold:

$\left(H_{\Psi}\right) \Psi: \mathcal{H} \rightarrow[0,+\infty)$ is convex, (Fréchet) differentiable with Lipschitz continuous gradient and $\operatorname{argmin} \Psi=\Psi^{-1}(0) \neq \emptyset$;

$\left(H_{\Theta}\right) \Theta: \mathcal{H} \rightarrow \mathbb{R}$ is convex, (Fréchet) differentiable with Lipschitz continuous gradient;

$\left(H_{\Phi}\right) \Phi: \mathcal{H} \rightarrow \mathbb{R} \cup\{+\infty\}$ is convex, lower semicontinuous and fulfills the relation

$S:=\{z \in \operatorname{argmin} \Psi \cap \operatorname{dom} \Phi: \Phi(z)+\Theta(z) \leq \Phi(x)+\Theta(x) \forall x \in \operatorname{argmin} \Psi\} \neq \emptyset$.

Here, $\operatorname{dom} \Phi=\{x \in \mathcal{H}: \Phi(x)<+\infty\}$ denotes the effective domain of the function $\Phi$.

In connection with (13), we investigate the nonautonomous dynamical system

$$
\left\{\begin{array}{l}
v(t) \in \partial \Phi(x(t)) \\
\lambda(t) \dot{x}(t)+\dot{v}(t)+v(t)+\nabla \Theta(x(t))+\beta(t) \nabla \Psi(x(t))=0 \\
x(0)=x_{0}, v(0)=v_{0} \in \partial \Phi\left(x_{0}\right),
\end{array}\right.
$$

where $x_{0}, v_{0} \in \mathcal{H}$ and

$$
\partial \Phi: \mathcal{H} \rightrightarrows \mathcal{H}, \partial \Phi(x):=\{p \in \mathcal{H}: \Phi(y) \geq \Phi(x)+\langle p, y-x\rangle \forall y \in \mathcal{H}\},
$$

for $\Phi(x) \in \mathbb{R}$ and $\partial \Phi(x):=\emptyset$ for $\Phi(x) \notin \mathbb{R}$, denotes the convex subdifferential of $\Phi$. We denote by $\operatorname{dom} \partial \Phi=\{x \in \mathcal{H}: \partial \Phi(x) \neq \emptyset\}$ the domain of the operator $\partial \Phi$. 
Furthermore, we make the following assumptions regarding the functions of time controlling the velocity and the penalty:

$$
\begin{aligned}
& \left(H_{\lambda}^{1}\right) \lambda:[0,+\infty) \rightarrow(0,+\infty) \text { is locally absolutely continuous; } \\
& \left(H_{\beta}^{1}\right) \beta:[0,+\infty) \rightarrow[0,+\infty) \text { is locally integrable. }
\end{aligned}
$$

Let us mention that due to $\left(H_{\lambda}^{1}\right), \dot{\lambda}(t)$ exists for almost every $t \geq 0$.

Remark 5 (a) In case $\Phi(x)=0$ for all $x \in \mathcal{H}$, the dynamical system (14) becomes

$$
\left\{\begin{array}{l}
\lambda(t) \dot{x}(t)+\nabla \Theta(x(t))+\beta(t) \nabla \Psi(x(t))=0 \\
x(0)=x_{0}
\end{array}\right.
$$

The asymptotic convergence of the trajectories generated by (15) has been investigated in [5] under the assumption $\lambda(t)=1$ for all $t \geq 0$, for $\Theta$ and $\Psi$ nonsmooth functions, by replacing their gradients with convex subdifferentials and, consequently, by treating the differential equation as a monotone inclusion (see (9)).

(b) In case $\Psi(x)=0$ for all $x \in \mathcal{H}$, the dynamical system

$$
\left\{\begin{array}{l}
v(t) \in \partial \Phi(x(t)) \\
\lambda(t) \dot{x}(t)+\dot{v}(t)+v(t)+\nabla \Theta(x(t))=0 \\
x(0)=x_{0}, v(0)=v_{0} \in \partial \Phi\left(x_{0}\right),
\end{array}\right.
$$

has been investigated in [2] (see, also, [10], for the situation when $\Theta(x)=0$ for all $x \in \mathcal{H})$.

(c) In case $\Theta(x)=0$ and $\Psi(x)=\frac{1}{2}\|x\|^{2}$ for all $x \in \mathcal{H}$ and $\lambda(t)=\lambda \in \mathbb{R}$ for every $t \in[0,+\infty)$, the Levenberg-Marquardt dynamical system (14) becomes

$$
\left\{\begin{array}{l}
v(t) \in \partial \Phi(x(t)) \\
\lambda \dot{x}(t)+\dot{v}(t)+v(t)+\beta(t) x(t)=0 \\
x(0)=x_{0}, v(0)=v_{0} \in \partial \Phi\left(x_{0}\right) .
\end{array}\right.
$$

The dynamical system (17) has been considered in [1] in connection with the problem of finding the minimal norm elements among the minima of $\Phi$, namely, (see also [4] and [9, Section 3])

$$
\inf _{x \in \operatorname{argmin} \Phi}\|x\|^{2} .
$$

In contrast to (14), where the function describing the constrained set of (13) is penalized, in (17) the objective function of (18) is penalized via a vanishing penalization function (see [1]).

In the following we specify what we understand under a solution of the dynamical system (14).

Definition 2 We say that the pair $(x, v)$ is a strong global solution of $(14)$, if the following properties are satisfied:

(i) $\quad x, v:[0,+\infty) \rightarrow \mathcal{H}$ are locally absolutely continuous functions;

(ii) $v(t) \in \partial \Phi(x(t))$ for every $t \in[0,+\infty)$;

(iii) $\lambda(t) \dot{x}(t)+\dot{v}(t)+v(t)+\nabla \Theta(x(t))+\beta(t) \nabla \Psi(x(t))=0$ for almost every $t \in[0,+\infty)$;

(iv) $x(0)=x_{0}, v(0)=v_{0}$. 
Similarly to the techniques used in [10], we will show the existence and uniqueness of the trajectories generated by (14) by converting it to an equivalent first order differential equation with respect to $z(\cdot)$, defined by

$$
z(t)=x(t)+\mu(t) v(t),
$$

where

$$
\mu(t)=\frac{1}{\lambda(t)} \forall t \geq 0 .
$$

To this end we will make use of the resolvent and Yosida approximation of the convex subdifferential of $\Psi$. For $\gamma>0$, we denote by

$$
J_{\gamma \partial \Phi}=(\operatorname{Id}+\gamma \partial \Phi)^{-1}
$$

the resolvent of $\gamma \partial \Phi$. Due to the maximal monotonicity of $\partial \Phi$, the resolvent $J_{\gamma \partial \Phi}: \mathcal{H} \rightarrow$ $\mathcal{H}$ is a single-valued operator with full-domain, which is, furthermore, nonexpansive, that is 1-Lipschitz continuous. Let us notice that the resolvent of the convex subdifferential is nothing else than the the proximal point operator and for all $x \in \mathcal{H}$ we have

$$
J_{\gamma \partial \Phi}(x)=\operatorname{prox}_{\gamma \Phi}(x)=\underset{u \in \mathcal{H}}{\operatorname{argmin}}\left\{\Phi(u)+\frac{1}{2 \gamma}\|u-x\|^{2}\right\} .
$$

The Yosida regularization of $\partial \Phi$ is defined by

$$
(\partial \Phi)_{\gamma}=\frac{1}{\gamma}\left(\mathrm{Id}-J_{\gamma \partial \Phi}\right)
$$

and it is $\gamma^{-1}$-Lipschitz continuous. For more properties of these operators we refer the reader to [11].

Assume now that $(x, v)$ is a strong global solution of (14). From (19) we have for every $t \in[0,+\infty)$

$$
v(t) \in \partial \Phi(x(t)) \Leftrightarrow z(t) \in x(t)+\mu(t) \partial \Phi(x(t))=(\operatorname{Id}+\mu(t) \partial \Phi)(x(t)),
$$

thus, from the definition of the resolvent we derive that relation (ii) in Definition 2 is equivalent to

$$
x(t)=J_{\mu(t) \partial \Phi}(z(t)) .
$$

From (19), (20) and the definition of the Yosida regularization we obtain

$$
v(t)=(\partial \Phi)_{\mu(t)}(z(t)) .
$$

Further, by differentiating (19) and taking into account (iii) in Definition 2, we get for almost every $t \in[0,+\infty)$

$\dot{z}(t)=\dot{x}(t)+\dot{\mu}(t) v(t)+\mu(t) \dot{v}(t)=\dot{\mu}(t) v(t)-\mu(t) v(t)-\mu(t) \nabla \Theta(x(t))-\beta(t) \mu(t) \nabla \Psi(x(t))$.

Taking into account (20), (21) and (22) we conclude that $z$ defined in (19) is a strong global solution of the dynamical system

$$
\left\{\begin{array}{l}
\dot{z}(t)+(\mu(t)-\dot{\mu}(t))(\partial \Phi)_{\mu(t)}(z(t))+\mu(t) \nabla \Theta\left(J_{\mu(t) \partial \Phi}(z(t))\right)+\beta(t) \mu(t) \nabla \Psi\left(J_{\mu(t) \partial \Phi}(z(t))\right)=0 \\
z(0)=x_{0}+\mu(0) v_{0} .
\end{array}\right.
$$

Vice versa, if $z$ is a strong global solution of (23), then one obtains via (20) and (21) a strong global solution of (14). 
Remark 6 By considering the time discretization $\dot{z}(t) \approx \frac{z_{n+1}-z_{n}}{h_{n}}$ of the above dynamical system and by taking $\mu$ constant, from (20) and (23) we obtain the iterative scheme

$$
(\forall n \geq 0)\left\{\begin{array}{l}
x_{n}=\operatorname{prox}_{\mu \Phi}\left(z_{n}\right) \\
z_{n+1}=\left(1-h_{n}\right) z_{n}+h_{n}\left(x_{n}-\mu \nabla \Theta\left(x_{n}\right)-\mu \beta_{n} \nabla \Psi\left(x_{n}\right)\right),
\end{array}\right.
$$

which for $h_{n}=1$ yields the following algorithm

$$
(\forall n \geq 0) x_{n+1}=\operatorname{prox}_{\mu \Phi}\left(x_{n}-\mu \nabla \Theta\left(x_{n}\right)-\mu \beta_{n} \nabla \Psi\left(x_{n}\right)\right) .
$$

The convergence of the above algorithm has been investigated in [16] in the more general framework of monotone inclusion problems, under the use of variable step sizes $\left(\mu_{n}\right)_{n \geq 0}$ and by assuming that

$$
\forall p \in \operatorname{ran} N_{\operatorname{argmin}} \Psi \sum_{n \in \mathbb{N}} \mu_{n} \beta_{n}\left[\Psi^{*}\left(\frac{p}{\beta_{n}}\right)-\sigma_{\operatorname{argmin}} \Psi\left(\frac{p}{\beta_{n}}\right)\right]<+\infty,
$$

which is a condition that can be seen as a discretized version of the one stated in (7). The case $\Theta(x)=0$ for all $x \in \mathcal{H}$ has been treated in [8] (see also the references therein).

Next we show that, given $x_{0}, v_{0} \in \mathcal{H}$ and by assuming $\left(H_{\lambda}^{1}\right)$ and $\left(H_{\beta}^{1}\right)$, there exists a unique strong global solution of the dynamical system (23). This will be done in the framework of the Cauchy-Lipschitz Theorem for absolutely continuous trajectories (see for example [19, Proposition 6.2.1], [22, Theorem 54]). To this end we will make use of the following Lipschitz property of the resolvent operator as a function of the step size, which actually is a consequence of the classical results [18, Proposition 2.6] and [11, Proposition 23.28] (see also [10, Proposition 2.3] and [2, Proposition 3.1]).

Proposition 7 Assume that $\left(H_{\Phi}\right)$ holds, $x \in \mathcal{H}$ and $0<\delta<+\infty$. Then the mapping $\tau \mapsto J_{\tau \partial \Phi} x$ is Lipschitz continuous on $[\delta,+\infty)$. More precisely, for any $\lambda_{1}, \lambda_{2} \in[\delta,+\infty)$ the following inequality holds:

$$
\left\|J_{\lambda_{1} \partial \Phi} x-J_{\lambda_{2} \partial \Phi} x\right\| \leq\left|\lambda_{1}-\lambda_{2}\right| \cdot\left\|(\partial \Phi)_{\delta} x\right\| .
$$

Furthermore, the function $\lambda \mapsto\left\|(\partial \Phi)_{\lambda} x\right\|$ is nonincreasing.

Notice that the dynamical system (23) can be written as

$$
\left\{\begin{array}{l}
\dot{z}(t)=f(t, z(t)) \\
z(0)=z_{0}
\end{array}\right.
$$

where $z_{0}=x_{0}+\mu(0) v_{0}$ and $f:[0,+\infty) \times \mathcal{H} \rightarrow \mathcal{H}$ is defined by

$$
f(t, w)=(\dot{\mu}(t)-\mu(t))(\partial \Phi)_{\mu(t)}(w)-\mu(t) \nabla \Theta\left(J_{\mu(t) \partial \Phi}(w)\right)-\beta(t) \mu(t) \nabla \Psi\left(J_{\mu(t) \partial \Phi}(w)\right) .
$$

In the following we denote by $L_{\nabla \Phi}$ and $L_{\nabla \Psi}$ the Lipschitz constants of $\nabla \Phi$ and $\nabla \Psi$, respectively.

(a) Notice that for every $t \geq 0$ and every $w_{1}, w_{2} \in \mathcal{H}$ we have

$$
\left\|f\left(t, w_{1}\right)-f\left(t, w_{2}\right)\right\| \leq\left(1+\frac{|\dot{\lambda}(t)|}{\lambda(t)}+\frac{L_{\nabla \Theta}}{\lambda(t)}+L_{\nabla \Psi} \frac{\beta(t)}{\lambda(t)}\right)\left\|w_{1}-w_{2}\right\| .
$$


Indeed, this follows (28), the Lipschitz properties of the operators involved and the definition of $\mu(t)$. Further, notice that due to $\left(H_{\lambda}^{1}\right)$ and $\left(H_{\beta}^{1}\right)$,

$$
L_{f}:[0,+\infty) \rightarrow \mathbb{R}, L_{f}(t)=1+\frac{|\dot{\lambda}(t)|}{\lambda(t)}+\frac{L_{\nabla \Theta}}{\lambda(t)}+L_{\nabla \Psi} \frac{\beta(t)}{\lambda(t)},
$$

which is for every $t \geq 0$ equal to the Lipschitz-constant of $f(t, \cdot)$, satisfies

$$
L_{f}(\cdot) \in L^{1}([0, b]) \text { for any } 0<b<+\infty .
$$

(b) We show now that

$$
\forall w \in \mathcal{H}, \forall b>0, \quad f(\cdot, w) \in L^{1}([0, b], \mathcal{H}) .
$$

We fix $w \in \mathcal{H}$ and $b>0$. Due to $\left(H_{\lambda}^{1}\right)$, there exist $\lambda_{\min }, \lambda_{\max }>0$ such that

$$
0<\lambda_{\min } \leq \lambda(t) \leq \lambda_{\max } \forall t \in[0, b]
$$

hence

$$
0<\frac{1}{\lambda_{\max }} \leq \mu(t) \leq \frac{1}{\lambda_{\min }} \forall t \in[0, b] .
$$

Relying on Proposition 7 we obtain for all $t \in[0, b]$ the following chain of inequalities:

$$
\begin{aligned}
\|f(t, w)\| \leq & |\dot{\mu}(t)-\mu(t)| \cdot\left\|(\partial \Phi) \frac{1}{\lambda_{\max }}(w)\right\| \\
& +\mu(t)\left\|\nabla \Theta\left(J_{\mu(t) \partial \Phi(w))-\nabla \Theta\left(J_{\frac{1}{\lambda_{\max }} \partial \Phi}(w)\right)\|+\mu(t)\| \nabla \Theta\left(J \frac{1}{\lambda_{\max }} \partial \Phi\right.}(w)\right)\right\| \\
& +\beta(t) \mu(t)\left\|\nabla \Psi\left(J_{\mu(t) \partial \Phi}(w)\right)-\nabla \Psi\left(J_{\frac{1}{\lambda_{\max }} \partial \Phi}(w)\right)\right\|+\beta(t) \mu(t)\left\|\nabla \Psi\left(J_{\frac{1}{\lambda_{\max }} \partial \Phi}(w)\right)\right\| \\
\leq & |\dot{\mu}(t)-\mu(t)| \cdot\left\|(\partial \Phi) \frac{1}{\lambda_{\max }}(w)\right\| \\
& +L_{\nabla \Theta} \mu(t)\left(\mu(t)-\frac{1}{\lambda_{\max }}\right) \cdot\left\|\nabla \Theta\left(J_{\frac{1}{\lambda_{\max }} \partial \Phi}(w)\right)\right\|+\mu(t)\left\|\nabla \Theta\left(J_{\frac{1}{\lambda_{\max }} \partial \Phi}(w)\right)\right\| \\
& +L_{\nabla \Psi} \beta(t) \mu(t)\left(\mu(t)-\frac{1}{\lambda_{\max }}\right) \cdot\left\|\nabla \Psi\left(J_{\frac{1}{\lambda_{\max }}} \partial \Phi(w)\right)\right\|+\beta(t) \mu(t)\left\|\nabla \Psi\left(J_{\frac{1}{\lambda_{\max }} \partial \Phi}(w)\right)\right\| .
\end{aligned}
$$

Now (30) follows from the properties of the functions $\mu$ and $\beta$, and the fact that

$$
|\dot{\mu}(t)-\mu(t)| \leq \frac{1}{\lambda_{\min }}\left(1+\frac{|\dot{\lambda}(t)|}{\lambda_{\min }}\right) \text { for almost every } t \geq 0 .
$$

In the light of the statements proven in (a) and (b), the existence and uniqueness of a strong global solution of the dynamical system (23) follow from [19, Proposition 6.2.1] (see also [22, Theorem 54]).

Finally, similarly to the proof of [10, Theorem 2.4(ii)], one can guarantee the existence and uniqueness of the trajectories generated by (14) by relying on the properties of the dynamical system (23) and on (20) and (21). The details are left to the reader.

\section{Convergence of the Trajectories and of the Objective Function Values}

In this section we prove weak convergence for the trajectory generated by the dynamical system (14) to an optimal solution of (13) as well as convergence for the objective function values of the latter along the trajectory. Some techniques from [5] and [10] will be useful in this context. 
To this end we will make the following supplementary assumptions:

$\left(H_{\lambda}^{2}\right) \quad \lambda:[0,+\infty) \rightarrow(0,+\infty)$ is locally absolutely continuous and $\dot{\lambda}(t) \leq 0$ for almost every $t \in[0,+\infty)$

$\left(H_{\beta}^{2}\right) \quad \beta:[0,+\infty) \rightarrow(0,+\infty)$ is measurable and bounded from above on each interval $[0, b]$, $0<b<+\infty$;

(H) $\forall p \in \operatorname{ran} N_{\operatorname{argmin}} \Psi \int_{0}^{+\infty} \beta(t)\left[\Psi^{*}\left(\frac{p}{\beta(t)}\right)-\sigma_{\operatorname{argmin}} \Psi\left(\frac{p}{\beta(t)}\right)\right] d t<+\infty$;

$(\widetilde{H}) \quad \partial\left(\Phi+\Theta+\delta_{\operatorname{argmin}} \Psi\right)=\partial \Phi+\nabla \Theta+N_{\operatorname{argmin}} \Psi$,

where

- $\quad N_{\operatorname{argmin}} \Psi$ is the normal cone to the set $\operatorname{argmin} \Psi: N_{\operatorname{argmin}} \Psi(x)=\{p \in \mathcal{H}:\langle p, y-x\rangle \leq$ $0 \forall y \in \operatorname{argmin} \Psi\}$ for $x \in \operatorname{argmin} \Psi$ and $N_{\operatorname{argmin}} \Psi(x)=\emptyset$ for $x \notin \operatorname{argmin} \Psi$;

- $\operatorname{ran} N_{\operatorname{argmin} \Psi}$ is the range of the normal cone $N_{\operatorname{argmin} \Psi}: p \in \operatorname{ran} N_{\operatorname{argmin}} \psi$ if and only if there exists $x \in \operatorname{argmin} \Psi$ such that $p \in N_{\operatorname{argmin}} \Psi(x)$;

- $\Psi^{*}: \mathcal{H} \rightarrow \mathbb{R} \cup\{+\infty\}$ is the Fenchel conjugate of $\Psi: \Psi^{*}(p)=\sup _{x \in \mathcal{H}}\{\langle p, x\rangle-$ $\Psi(x)\} \forall p \in \mathcal{H}$;

- $\sigma_{\operatorname{argmin}} \Psi: \mathcal{H} \rightarrow \mathbb{R} \cup\{+\infty\}$ is the support function of the set $\operatorname{argmin} \Psi: \sigma_{\operatorname{argmin}} \Psi(p)=$ $\sup _{x \in \operatorname{argmin} \Psi}\langle p, x\rangle$ for all $p \in \mathcal{H}$;

- $\delta_{\operatorname{argmin}} \Psi: \mathcal{H} \rightarrow \mathbb{R} \cup\{+\infty\}$ is the indicator function of $\operatorname{argmin} \Psi$ : it takes the value 0 on the set $\operatorname{argmin} \Psi$ and $+\infty$, otherwise.

We have $N_{\operatorname{argmin}} \Psi=\partial \delta_{\operatorname{argmin}} \Psi$. Moreover, $p \in N_{\operatorname{argmin}} \Psi(x)$ if and only if $x \in \operatorname{argmin} \Psi$ and $\sigma_{\operatorname{argmin}} \Psi(p)=\langle p, x\rangle$.

Remark 8 (a) The condition $\dot{\lambda}(t) \leq 0$ for almost every $t \in[0,+\infty)$ has been used in [10] in the study of the asymptotic convergence of the dynamical system (1), when approaching the monotone inclusion problem (2).

(b) Under $\left(H_{\Psi}\right)$, due to $\Psi \leq \delta_{\operatorname{argmin}} \Psi$, we have $\Psi^{*} \geq \delta_{\operatorname{argmin} \Psi}^{*}=\sigma_{\operatorname{argmin}} \Psi$.

(c) When $\Psi=0$ (see Remark $5(\mathrm{~b})$ ), it holds $N_{\operatorname{argmin}} \Psi(x)=\{0\}$ for every $x \in$ $\operatorname{argmin} \Psi=\mathcal{H}, \Psi^{*}=\sigma_{\operatorname{argmin}} \Psi=\delta_{\{0\}}$, which shows that in this case $(H)$ trivially holds.

(d) A nontrivial situation in which condition $(H)$ is fulfilled is when $\psi(x)=\frac{1}{2} \inf _{y \in C}$ $\|x-y\|^{2}$, for a nonempty, convex and closed set $C \subseteq \mathcal{H}$ (see [5]). Then (7) holds if and only if

$$
\int_{0}^{+\infty} \frac{1}{\beta(t)} d t<+\infty
$$

which is trivially satisfied for $\beta(t)=(1+t)^{\alpha}$ with $\alpha>1$.

(e) Due to the continuity of $\Theta$, the condition $(\widetilde{H})$ is equivalent to

$$
\partial\left(\Phi+\delta_{\operatorname{argmin}} \Psi\right)=\partial \Phi+N_{\operatorname{argmin}} \Psi,
$$

which holds when $0 \in \operatorname{sqri}(\operatorname{dom} \Phi-\operatorname{argmin} \Psi)$, a condition that is fulfilled, if $\Phi$ is continuous at a point in $\operatorname{dom} \Phi \cap \operatorname{argmin} \Psi \operatorname{or} \operatorname{int}(\operatorname{argmin} \Psi) \cap \operatorname{dom} \Phi \neq \emptyset$ (we invite the reader to consult also $[11,13]$ and [23] for other sufficient conditions for the above subdifferential sum formula). Here, for $M \subseteq \mathcal{H}$ a convex set,

$$
\text { sqri } M:=\left\{x \in M: \cup_{\lambda>0} \lambda(M-x) \text { is a closed linear subspace of } \mathcal{H}\right\}
$$

denotes its strong quasi-relative interior. We always have int $M \subseteq$ sqri $M$ (in general this inclusion may be strict). If $\mathcal{H}$ is finite-dimensional, then sqri $M$ coincides with 
ri $M$, the relative interior of $M$, which is the interior of $M$ with respect to its affine hull.

The following differentiability result of the composition of convex functions with absolutely continuous trajectories that is due to Brézis (see [18, Lemme 4, p. 73] and also [5, Lemma 3.2]) will play an important role in our analysis.

Lemma 9 Let $f: \mathcal{H} \rightarrow \mathbb{R} \cup\{+\infty\}$ be a proper, convex and lower semicontinuous function. Let $x \in L^{2}([0, T], \mathcal{H})$ be absolutely continuous such that $\dot{x} \in L^{2}([0, T], \mathcal{H})$ and $x(t) \in$ $\operatorname{dom} f$ for almost every $t \in[0, T]$. Assume that there exists $\xi \in L^{2}([0, T], \mathcal{H})$ such that $\xi(t) \in \partial f(x(t))$ for almost every $t \in[0, T]$. Then the function $t \mapsto f(x(t))$ is absolutely continuous and for every $t$ such that $x(t) \in \operatorname{dom} \partial f$ we have

$$
\frac{d}{d t} f(x(t))=\langle\dot{x}(t), h\rangle \forall h \in \partial f(x(t)) .
$$

We start our convergence analysis with the following technical result.

Lemma 10 Assume that $\left(H_{\Psi}\right),\left(H_{\Theta}\right),\left(H_{\Phi}\right),\left(H_{\lambda}^{1}\right)$ and $\left(H_{\beta}^{2}\right)$ hold and let $(x, v)$ : $[0,+\infty) \rightarrow \mathcal{H} \times \mathcal{H}$ be a strong stable solution of the dynamical system (14). Then the following statements are true:

(i) $\langle\dot{x}(t), \dot{v}(t)\rangle \geq 0$ for almost every $t \in[0,+\infty)$;

(ii) $\frac{d}{d t} \Phi(x(t))=\langle\dot{x}(t), v(t)\rangle$ for almost every $t \in[0,+\infty)$.

Proof (i) See [10, Proposition 3.1]. The proof relies on the first relation in (14) and the monotonicity of the convex subdifferential.

(ii) The proof makes use of Lemma 9. Let $T>0$ be fixed. Due to the continuity of $x$ and $v$ we obviously have

$$
x, v \in L^{2}([0, T], \mathcal{H}) .
$$

The only condition which has to be checked is $\dot{x} \in L^{2}([0, T], \mathcal{H})$. By considering the second relation in (14) and by inner multiplying it with $\dot{x}(t)$, we derive for almost every $t \in[0, T]$

$\lambda(t)\|\dot{x}(t)\|^{2}+\langle\dot{x}(t), \dot{v}(t)\rangle+\langle\dot{x}(t), v(t)\rangle+\langle\dot{x}(t), \nabla \Theta(x(t))\rangle+\beta(t)\langle\dot{x}(t), \nabla \Psi(x(t))\rangle=0$.

Using (i) we obtain for almost every $t \in[0, T]$

$$
\lambda(t)\|\dot{x}(t)\|^{2}+\langle\dot{x}(t), v(t)\rangle+\langle\dot{x}(t), \nabla \Theta(x(t))\rangle+\beta(t)\langle\dot{x}(t), \nabla \Psi(x(t))\rangle \leq 0 .
$$

Since $x, v$ are continuous on $[0, T]$, they are bounded on $[0, T]$, a property which is shared also by $t \mapsto \beta(t) \nabla \Psi(x(t))$, due to $\left(H_{\beta}^{2}\right)$ and $\left(H_{\Psi}\right)$, and by $t \mapsto \nabla \Theta(x(t))$, due to $\left(H_{\Theta}\right)$. Since $\lambda$ is bounded from below by a positive constant on $[0, T]$, from (31) one easily obtains that

$$
\dot{x} \in L^{2}([0, T], \mathcal{H})
$$

and the conclusion follows by applying Lemma 9.

Lemma 11 Assume that $\left(H_{\Psi}\right),\left(H_{\Theta}\right),\left(H_{\Phi}\right),\left(H_{\lambda}^{2}\right),\left(H_{\beta}^{2}\right),(H)$ and $(\tilde{H})$ hold and let $(x, v)$ : $[0,+\infty) \rightarrow \mathcal{H} \times \mathcal{H}$ be a strong stable solution of the dynamical system (14). Choose 
arbitrary $z \in S$ and $p \in N_{\operatorname{argmin}} \Psi(z)$ such that $-p-\nabla \Theta(z) \in \partial \Phi(z)$. Define $g_{z}, h_{z}$ : $[0,+\infty) \rightarrow[0,+\infty)$ as

$$
g_{z}(t)=\Phi(z)-\Phi(x(t))+\langle v(t), x(t)-z\rangle
$$

and

$$
h_{z}(t)=\Theta(z)-\Theta(x(t))+\langle\nabla \Theta(x(t)), x(t)-z\rangle .
$$

The following statements are true:

(i) $\exists \lim _{t \rightarrow+\infty}\left(\frac{\lambda(t)}{2}\|x(t)-z\|^{2}+g_{z}(t)\right) \in[0,+\infty)$;

(ii) $\int_{0}^{+\infty} \beta(t) \Psi(x(t)) d t<+\infty$;

(iii) $\exists \lim _{t \rightarrow+\infty} \int_{0}^{t}\langle p, x(s)-z\rangle d s \in \mathbb{R}$;

(iv) $\exists \lim _{t \rightarrow+\infty} \int_{0}^{t}((\Phi+\Theta)(x(s))-(\Phi+\Theta)(z)+\beta(s) \Psi(x(s))) d s \in \mathbb{R}$;

(v) $\exists \lim _{t \rightarrow+\infty} \int_{0}^{t}(\langle v(s), x(s)-z\rangle+\langle\nabla \Theta(x(s)), x(s)-z\rangle+\beta(s) \Psi(x(s)) d s \in \mathbb{R}$;

(vi) $\exists \lim _{t \rightarrow+\infty} \int_{0}^{t}((\Phi+\Theta)(x(s))-(\Phi+\Theta)(z)) d s \in \mathbb{R}$;

(vii) $\exists \lim _{t \rightarrow+\infty} \int_{0}^{t}(\langle v(s), x(s)-z\rangle+\langle\nabla \Theta(x(s)), x(s)-z\rangle) d s \in \mathbb{R}$;

(viii) $g_{z} \in L^{1}([0,+\infty)) \cap L^{\infty}([0,+\infty))$ and $h_{z} \in L^{1}([0,+\infty))$.

Proof For the beginning, we notice that from the definition of $S$ and $(\tilde{H})$ we have

$$
0 \in \partial\left(\Phi+\Theta+\delta_{\operatorname{argmin}} \Psi\right)(z)=\partial \Phi(z)+\nabla \Theta(z)+N_{\operatorname{argmin}} \Psi(z),
$$

hence there exists

such that

$$
p \in N_{\operatorname{argmin}} \Psi(z)
$$

$$
-p-\nabla \Theta(z) \in \partial \Phi(z) .
$$

For almost every $t \geq 0$ it holds according to (14)

$$
\begin{aligned}
\frac{d}{d t}\left(\frac{\lambda(t)}{2}\|x(t)-z\|^{2}\right)= & \frac{\dot{\lambda}(t)}{2}\|x(t)-z\|^{2}+\lambda(t)\langle\dot{x}(t), x(t)-z\rangle \\
= & \frac{\dot{\lambda}(t)}{2}\|x(t)-z\|^{2}-\langle\dot{v}(t), x(t)-z\rangle-\langle v(t), x(t)-z\rangle \\
& -\langle\nabla \Theta(x(t)), x(t)-z\rangle-\beta(t)\langle\nabla \Psi(x(t)), x(t)-z\rangle .
\end{aligned}
$$

From (14) and the convexity of $\Phi, \Theta$ and $\Psi$ we have for every $t \in[0,+\infty)$

$$
\begin{gathered}
\Phi(z) \geq \Phi(x(t))+\langle v(t), z-x(t)\rangle \\
\Theta(z) \geq \Theta(x(t))+\langle\nabla \Theta(x(t)), z-x(t)\rangle
\end{gathered}
$$

and

$$
0=\Psi(z) \geq \Psi(x(t))+\langle\nabla \Psi(x(t)), z-x(t)\rangle .
$$

From (33) and the convexity $\Phi$ and $\Theta$ we obtain for every $t \in[0,+\infty)$

$$
\Phi(x(t)) \geq \Phi(z)+\langle-p-\nabla \Theta(z), x(t)-z\rangle
$$

and

$$
\Theta(x(t)) \geq \Theta(z)+\langle\nabla \Theta(z), x(t)-z\rangle .
$$

Further, due to Lemma 10(ii) it holds for almost every $t \in[0,+\infty)$

$$
\begin{aligned}
\frac{d}{d t} g_{z}(t) & =-\langle\dot{x}(t), v(t)\rangle+\langle\dot{v}(t), x(t)-z\rangle+\langle v(t), \dot{x}(t)\rangle \\
& =\langle\dot{v}(t), x(t)-z\rangle .
\end{aligned}
$$


On the other hand, using (32) and the Young-Fenchel inequality we obtain for every $t \in[0,+\infty)$

$$
\begin{aligned}
\beta(t) \Psi(x(t))+\langle-p, x(t)-z\rangle & =\beta(t)\left(\Psi(x(t))+\left\langle\frac{-p}{\beta(t)}, x(t)-z\right\rangle\right) \\
& =\beta(t)\left(\Psi(x(t))-\left\langle\frac{p}{\beta(t)}, x(t)\right\rangle+\sigma_{\operatorname{argmin}} \Psi\left(\frac{p}{\beta(t)}\right)\right) \\
& \geq \beta(t)\left(-\Psi^{*}\left(\frac{p}{\beta(t)}\right)+\sigma_{\operatorname{argmin}} \Psi\left(\frac{p}{\beta(t)}\right)\right) .
\end{aligned}
$$

Finally, we obtain for almost every $t \in[0,+\infty)$

$$
\begin{aligned}
& \frac{d}{d t}\left(\frac{\lambda(t)}{2}\|x(t)-z\|^{2}+g_{z}(t)\right)+\beta(t)\left(-\Psi^{*}\left(\frac{p}{\beta(t)}\right)+\sigma_{\operatorname{argmin}} \Psi\left(\frac{p}{\beta(t)}\right)\right) \\
\leq & \frac{d}{d t}\left(\frac{\lambda(t)}{2}\|x(t)-z\|^{2}+g_{z}(t)\right)+\beta(t) \Psi(x(t))+\langle-p, x(t)-z\rangle \\
\leq & \frac{d}{d t}\left(\frac{\lambda(t)}{2}\|x(t)-z\|^{2}+g_{z}(t)\right)+(\Phi+\Theta)(x(t))-(\Phi+\Theta)(z)+\beta(t) \Psi(x(t)) \\
\leq & \frac{d}{d t}\left(\frac{\lambda(t)}{2}\|x(t)-z\|^{2}+g_{z}(t)\right)+\langle v(t), x(t)-z\rangle+\langle\nabla \Theta(x(t)), x(t)-z\rangle+\beta(t) \Psi(x(t)) \\
\leq & 0,
\end{aligned}
$$

where the first inequality follows from (41), the second one from (38) and (39), the next one from (35) and (36), and the last one from $\left(H_{\lambda}^{2}\right),(34),(40)$ and (37).

(i) Since for almost every $t \in[0,+\infty)$ we have (see (42))

$$
\frac{d}{d t}\left(\frac{\lambda(t)}{2}\|x(t)-z\|^{2}+g_{z}(t)\right) \leq \beta(t)\left(\Psi^{*}\left(\frac{p}{\beta(t)}\right)-\sigma_{\operatorname{argmin}} \Psi\left(\frac{p}{\beta(t)}\right)\right),
$$

the conclusion follows from Lemma $2,(H)$ and the fact that $g_{z}(t) \geq 0$ for every $t \geq 0$.

(ii) Let $F:[0,+\infty) \rightarrow \mathbb{R}$ be defined by

$$
F(t)=\int_{0}^{t}(-\beta(s) \Psi(x(s))+\langle p, x(s)-z\rangle) d s \forall t \in[0,+\infty) .
$$

From (42) we have for almost every $s \in[0,+\infty)$

$$
-\beta(s) \Psi(x(s))+\langle p, x(s)-z\rangle \geq \frac{d}{d s}\left(\frac{\lambda(s)}{2}\|x(s)-z\|^{2}+g_{z}(s)\right) .
$$

By integration we obtain for every $t \in[0,+\infty)$

$$
\begin{aligned}
F(t) & \geq \frac{\lambda(t)}{2}\|x(t)-z\|^{2}+g_{z}(t)-\frac{\lambda(0)}{2}\left\|x_{0}-z\right\|^{2}-g_{z}(0) \\
& \geq-\frac{\lambda(0)}{2}\left\|x_{0}-z\right\|^{2}-g_{z}(0)
\end{aligned}
$$


hence $F$ is bounded from below. Furthermore, from (41) we derive for every $t \in[0,+\infty)$

$$
\begin{aligned}
\frac{d}{d t} F(t) & =-\beta(t) \Psi(x(t))+\langle p, x(t)-z\rangle \\
& \leq \beta(t)\left(\Psi^{*}\left(\frac{p}{\beta(t)}\right)-\sigma_{\operatorname{argmin}} \Psi\left(\frac{p}{\beta(t)}\right)\right) .
\end{aligned}
$$

From $(H)$ and Lemma 2 it follows that $\lim _{t \rightarrow+\infty} F(t)$ exists and it is a real number. Hence

$$
\exists \lim _{t \rightarrow+\infty} \int_{0}^{t}(\beta(s) \Psi(s)+\langle-p, x(s)-z\rangle) d s \in \mathbb{R} .
$$

Further, since $\psi \geq 0$, we obtain for every $t \in[0,+\infty)$

$$
\beta(t) \Psi(x(t))+\langle-p, x(t)-z\rangle \geq \frac{\beta(t)}{2} \Psi(x(t))+\langle-p, x(t)-z\rangle .
$$

Similarly to (41) one can show that for every $t \in[0,+\infty)$

$$
\frac{\beta(t)}{2} \Psi(x(t))+\langle-p, x(t)-z\rangle \geq \frac{\beta(t)}{2}\left(-\Psi^{*}\left(\frac{2 p}{\beta(t)}\right)+\sigma_{\operatorname{argmin}} \Psi\left(\frac{2 p}{\beta(t)}\right)\right),
$$

while from (42) we obtain that for almost every $t \in[0,+\infty)$ it holds

$$
\begin{aligned}
& \frac{d}{d t}\left(\frac{\lambda(t)}{2}\|x(t)-z\|^{2}+g_{z}(t)\right)+\frac{\beta(t)}{2}\left(-\Psi^{*}\left(\frac{2 p}{\beta(t)}\right)+\sigma_{\operatorname{argmin}} \Psi\left(\frac{2 p}{\beta(t)}\right)\right) \\
\leq & \frac{d}{d t}\left(\frac{\lambda(t)}{2}\|x(t)-z\|^{2}+g_{z}(t)\right)+\frac{\beta(t)}{2} \Psi(x(t))+\langle-p, x(t)-z\rangle \\
\leq & \frac{d}{d t}\left(\frac{\lambda(t)}{2}\|x(t)-z\|^{2}+g_{z}(t)\right)+\beta(t) \Psi(x(t))+\langle-p, x(t)-z\rangle \\
\leq & 0
\end{aligned}
$$

By using the same arguments as used in the proof of (43) it yields that

$$
\exists \lim _{t \rightarrow+\infty} \int_{0}^{t}\left(\frac{\beta(s)}{2} \Psi(s)+\langle-p, x(s)-z\rangle\right) d s \in \mathbb{R} .
$$

Finally, from (43) and (44) we obtain (ii).

(iii) Follows from (43) and (ii).

(iv)-(v) These statements follow from (42) and (41), by using similar arguments as used for proving (43).

(vi)-(vii) These statements are direct consequences of (iv), (v) and (ii).

(viii) Combining (vi) and (vii) with $g_{z}, h_{z} \geq 0$, we easily derive that

$$
g_{z}+h_{z} \in L^{1}([0,+\infty)) \text {. }
$$

Since

$$
0 \leq g_{z} \leq g_{z}+h_{z},
$$

we deduce that $g_{z} \in L^{1}([0,+\infty))$ and $h_{z} \in L^{1}([0,+\infty))$. Finally, notice that due to (i) there exists $T>0$ such that $g_{z}$ is bounded on $[T,+\infty)$. The boundedness of $g_{z}$ on $[0, T]$ follows from (38) and the continuity of $x$ and $v$. Thus, $g_{z} \in L^{\infty}([0,+\infty))$. 
In order to proceed with the asymptotic analysis of the dynamical system (14), we make the following more involved assumptions on the functions $\lambda$ and $\beta$, respectively:

$\left(H_{\lambda}^{3}\right) \quad \lambda:[0,+\infty) \rightarrow(0,+\infty)$ is locally absolutely continuous, $\dot{\lambda}(t) \leq 0$ for almost every $t \in[0,+\infty)$ and $\lim _{t \rightarrow+\infty} \lambda(t)>0 ;$

$\left(H_{\beta}^{3}\right) \quad \beta:[0,+\infty) \rightarrow(0,+\infty)$ is locally absolutely continuous, it satisfies for some $k \geq 0$ the growth condition $0 \leq \dot{\beta}(t) \leq k \beta(t)$ for almost every $t \in[0,+\infty)$ and $\lim _{t \rightarrow+\infty} \beta(t)=+\infty$.

Lemma 12 Assume that $\left(H_{\Psi}\right),\left(H_{\Theta}\right),\left(H_{\Phi}\right),\left(H_{\lambda}^{3}\right),\left(H_{\beta}^{3}\right),(H)$ and $(\widetilde{H})$ hold and let $(x, v)$ : $[0,+\infty) \rightarrow \mathcal{H} \times \mathcal{H}$ be a strong stable solution of the dynamical system (14). The following statements are true:

(i) $x$ is bounded;

(ii) $\lim _{t \rightarrow+\infty} \Psi(x(t))=0$.

Proof Take an arbitrary $z \in S$ and (according to $(\tilde{H})) p \in N_{\operatorname{argmin} \Psi}(z)$ such that $-p-$ $\nabla \Theta(z) \in \partial \Phi(z)$ and consider the functions $g_{z}, h_{z}$ defined in Lemma 11.

(i) According to Lemma 11(i), since $g_{z} \geq 0$, we have that $t \mapsto \lambda(t)\|x(t)-z\|^{2}$ is bounded, which combined with $\lim _{t \rightarrow+\infty} \lambda(t)>0$ implies that $x$ is bounded.

(ii) Consider the function $E_{1}:[0,+\infty) \rightarrow \mathbb{R}$ defined for every $t \in[0,+\infty)$ by

$$
E_{1}(t)=\frac{(\Phi+\Theta)(x(t))}{\beta(t)}+\Psi(x(t)) .
$$

Using Lemma 10 and (14) we obtain for almost every $t \in[0,+\infty)$

$$
\begin{aligned}
\dot{E}_{1}(t)= & \frac{1}{\beta(t)}(\langle v(t), \dot{x}(t)\rangle+\langle\nabla \Theta(x(t)), \dot{x}(t)\rangle)-\frac{\dot{\beta}(t)}{\beta^{2}(t)}(\Phi+\Theta)(x(t)) \\
& +\langle\nabla \Psi(x(t)), \dot{x}(t)\rangle \\
= & \frac{1}{\beta(t)}\langle v(t)+\nabla \Theta(x(t))+\beta(t) \nabla \Psi(x(t)), \dot{x}(t)\rangle-\frac{\dot{\beta}(t)}{\beta^{2}(t)}(\Phi+\Theta)(x(t)) \\
= & \frac{1}{\beta(t)}\langle-\lambda(t) \dot{x}(t)-\dot{v}(t), \dot{x}(t)\rangle-\frac{\dot{\beta}(t)}{\beta^{2}(t)}(\Phi+\Theta)(x(t)) \\
= & -\frac{\lambda(t)}{\beta(t)}\|\dot{x}(t)\|^{2}-\frac{1}{\beta(t)}\langle\dot{v}(t), \dot{x}(t)\rangle-\frac{\dot{\beta}(t)}{\beta^{2}(t)}(\Phi+\Theta)(x(t)) \\
\leq & -\frac{\dot{\beta}(t)}{\beta^{2}(t)} \inf _{t \geq 0}(\Phi+\Theta)(x(t)),
\end{aligned}
$$

where we used that, according to (38), (39) and (i), $(\Phi+\Theta)(x(t))$ is bounded from below. From (45) and Lemma 2 it follows that there exists $\lim _{t \rightarrow+\infty} E_{1}(t) \in \mathbb{R}$.

Using now Lemma 11(iv) we get

$$
\liminf _{t \rightarrow+\infty}((\Phi+\Theta)(x(t))-(\Phi+\Theta)(z)+\beta(t) \Psi(x(t))) \leq 0
$$


and, since $(\Phi+\Theta)(x(t))$ is bounded from below, this limes inferior is a real number. Let $\left(t_{n}\right)_{n \in \mathbb{N}}$ be a sequence with $\lim _{n \rightarrow+\infty} t_{n}=+\infty$ such that

$$
\begin{aligned}
& \lim _{n \rightarrow+\infty}\left((\Phi+\Theta)\left(x\left(t_{n}\right)\right)-(\Phi+\Theta)(z)+\beta\left(t_{n}\right) \Psi\left(x\left(t_{n}\right)\right)\right)= \\
& \liminf _{t \rightarrow+\infty}((\Phi+\Theta)(x(t))-(\Phi+\Theta)(z)+\beta(t) \Psi(x(t))) \in \mathbb{R} .
\end{aligned}
$$

Since

$E_{1}\left(t_{n}\right)=\frac{1}{\beta\left(t_{n}\right)}\left((\Phi+\Theta)\left(x\left(t_{n}\right)\right)-(\Phi+\Theta)(z)+\beta\left(t_{n}\right) \Psi\left(x\left(t_{n}\right)\right)\right)+\frac{(\Phi+\Theta)(z)}{\beta\left(t_{n}\right)} \forall n \in \mathbb{N}$ and $\lim _{n \rightarrow+\infty} \beta\left(t_{n}\right)=+\infty$, it yields that $\lim _{n \rightarrow+\infty} E_{1}\left(t_{n}\right)=0$. Thus, since $\lim _{t \rightarrow+\infty}$ $E_{1}(t)$ exists,

$$
\lim _{t \rightarrow+\infty} E_{1}(t)=0 .
$$

The statement follows by taking into consideration that for every $t \in[0,+\infty)$

$0 \leq \Psi(x(t)) \leq \Psi(x(t))+\frac{1}{\beta(t)}\left((\Phi+\Theta)(x(t))-\inf _{s \geq 0}(\Phi+\Theta)(x(s))\right)=E_{1}(t)-\frac{1}{\beta(t)} \inf _{s \geq 0}(\Phi+\Theta)(x(s))$ in combination with $\lim _{t \rightarrow+\infty} \beta(t)=+\infty$.

Lemma 13 Assume that $\left(H_{\Psi}\right),\left(H_{\Theta}\right),\left(H_{\Phi}\right),\left(H_{\lambda}^{3}\right),\left(H_{\beta}^{3}\right),(H)$ and $(\widetilde{H})$ hold and let $(x, v)$ : $[0,+\infty) \rightarrow \mathcal{H} \times \mathcal{H}$ be a strong stable solution of the dynamical system (14). Then

$$
\liminf _{t \rightarrow+\infty}(\Phi+\Theta)(x(t)) \geq(\Phi+\Theta)(z) \forall z \in S .
$$

Proof Take an arbitrary $z \in S$. From $(\tilde{H})$ there exists $p \in N_{\operatorname{argmin}} \Psi(z)$ such that $-p-$ $\nabla \Theta(z) \in \partial \Phi(z)$. From Lemma 11(iii) we get

$$
\liminf _{t \rightarrow+\infty}\langle-p, x(t)-z\rangle \leq 0 .
$$

We claim that

$$
\liminf _{t \rightarrow+\infty}\langle-p, x(t)-z\rangle=0 .
$$

Since according to the previous lemma $x$ is bounded, this limit inferior is a real number. Let $\left(t_{n}\right)_{n \in \mathbb{N}}$ be a sequence with $\lim _{n \rightarrow+\infty} t_{n}=+\infty$ such that

$$
\lim _{n \rightarrow+\infty}\left\langle-p, x\left(t_{n}\right)-z\right\rangle=\liminf _{t \rightarrow+\infty}\langle-p, x(t)-z\rangle \in \mathbb{R} .
$$

Using again that $x$ is bounded, there exists $\bar{x} \in \mathcal{H}$ and a subsequence $\left(x\left(t_{n_{k}}\right)\right)$ such that $\left(x\left(t_{n_{k}}\right)\right)_{k \geq 0}$ converges weakly to $\bar{x}$ as $k \rightarrow+\infty$. From (49) we derive

$$
\liminf _{t \rightarrow+\infty}\langle-p, x(t)-z\rangle=\langle-p, \bar{x}-z\rangle .
$$

Since $\Psi$ is weak lower semicontinuous, from Lemma 12(ii) we get

$$
0 \leq \Psi(\bar{x}) \leq \liminf _{k \rightarrow+\infty} \Psi\left(x\left(t_{n_{k}}\right)\right)=0,
$$

hence $\bar{x} \in \operatorname{argmin} \Psi$. Combining this with $p \in N_{\operatorname{argmin}} \Psi(z)$ we derive $\langle-p, \bar{x}-z\rangle \geq 0$. From (50) and (47) we conclude that (48) is true. Moreover, due to $-p-\nabla \Theta(z) \in \partial \Phi(z)$, (38) and (39) we obtain

$$
(\Phi+\Theta)(x(t)) \geq(\Phi+\Theta)(z)+\langle-p, x(t)-z\rangle
$$

and the conclusion follows from (48). 
Remark 14 One can notice that the condition $\dot{\beta} \leq k \beta$ has not been used in the proofs of Lemma 12 and Lemma 13.

We come now to the main results of the paper.

Theorem 15 Assume that $\left(H_{\Psi}\right),\left(H_{\Theta}\right),\left(H_{\Phi}\right),\left(H_{\lambda}^{3}\right),\left(H_{\beta}^{3}\right),(H)$ and $(\tilde{H})$ hold and let $(x, v):[0,+\infty) \rightarrow \mathcal{H} \times \mathcal{H}$ be a strong stable solution of the dynamical system (14). The following statements are true:

(i) $\int_{0}^{+\infty} \beta(t) \Psi(x(t)) d t<+\infty$;

(ii) $\dot{x} \in L^{2}([0,+\infty) ; \mathcal{H})$;

(iii) $\langle\dot{x}, \dot{v}\rangle \in L^{1}([0,+\infty))$;

(iv) $(\Phi+\Theta)(x(t))$ converges to the optimal objective value of (13) as $t \rightarrow+\infty$;

(v) $\lim _{t \rightarrow+\infty} \Psi(x(t))=\lim _{t \rightarrow+\infty} \beta(t) \Psi(x(t))=0$;

(vi) $x(t)$ converges weakly to an optimal solution of (13) as $t \rightarrow+\infty$.

Proof Take an arbitrary $z \in S$. From $(\widetilde{H})$ there exists $p \in N_{\operatorname{argmin}} \Psi(z)$ such that $-p-$ $\nabla \Theta(z) \in \partial \Phi(z)$. Consider again the functions $g_{z}, h_{z}$ defined in Lemma 11.

Notice that statement (i) has been already proved in Lemma 11.

Further, consider the function $E_{2}:[0,+\infty) \rightarrow \mathbb{R}$ defined for every $t \in[0,+\infty)$ as

$$
E_{2}(t)=(\Phi+\Theta)(x(t))+\beta(t) \Psi(x(t)) .
$$

By using Lemma 10, relation (14) and $\left(H_{\beta}^{3}\right)$ we derive for almost every $t \in[0,+\infty)$

$$
\begin{aligned}
\dot{E}_{2}(t) & =\langle v(t), \dot{x}(t)\rangle+\langle\nabla \Theta(x(t)), \dot{x}(t)\rangle+\beta(t)\langle\nabla \Psi(x(t)), \dot{x}(t)\rangle+\dot{\beta}(t) \Psi(x(t)) \\
& =\langle v(t)+\nabla \Theta(x(t))+\beta(t) \nabla \Psi(x(t)), \dot{x}(t)\rangle+\dot{\beta}(t) \Psi(x(t)) \\
& =\langle-\lambda(t) \dot{x}(t)-\dot{v}(t), \dot{x}(t)\rangle+\dot{\beta}(t) \Psi(x(t)) \\
& \leq-\lambda(t)\|\dot{x}(t)\|^{2}-\langle\dot{x}(t), \dot{v}(t)\rangle+k \beta(t) \Psi(x(t)) .
\end{aligned}
$$

Since $E_{2}$ is bounded from below, a simple integration procedure in (51) combined with (i), Lemma 10(i) and Lemma 2 yields

$$
\begin{gathered}
\exists \lim _{t \rightarrow+\infty} E_{2}(t) \in \mathbb{R}, \\
\int_{0}^{+\infty} \lambda(t)\|\dot{x}(t)\|^{2} d t<+\infty
\end{gathered}
$$

and

$$
\int_{0}^{+\infty}\langle\dot{x}(t), \dot{v}(t)\rangle d t<+\infty
$$

which is statement (iii). Statement (ii) follows by taking into account that $\lim _{i n f} \rightarrow+\infty$ $\lambda(t)>0$.

Further, since $\beta(t) \Psi(x(t)) \geq 0$, from (46) and Lemma 13 we get that

$$
\liminf _{t \rightarrow+\infty}((\Phi+\Theta)(x(t))-(\Phi+\Theta)(z)+\beta(t) \Psi(x(t)))=0 .
$$

Taking into account the definition of $E_{2}$ and the fact that $\lim _{t \rightarrow+\infty} E_{2}(t) \in \mathbb{R}$, we conclude that

$$
\lim _{t \rightarrow+\infty} E_{2}(t)=(\Phi+\Theta)(z)
$$


Further, we have

$\limsup _{t \rightarrow+\infty}(\Phi+\Theta)(x(t)) \leq \limsup _{t \rightarrow+\infty}((\Phi+\Theta)(x(t))+\beta(t) \Psi(x(t)))=\lim _{t \rightarrow+\infty} E_{2}(t)=(\Phi+\Theta)(z)$, which combined with Lemma 13 yields

$$
\lim _{t \rightarrow+\infty}(\Phi+\Theta)(x(t))=(\Phi+\Theta)(z),
$$

hence (iv) holds.

The statement (v) is a consequence of Lemma 12(ii), (54), (55) and the definition of $E_{2}$.

In order to prove statement (vi), we will make use of the Opial Lemma 4. From (42) we have for almost every $t \in[0,+\infty)$

$\frac{\dot{\lambda}(t)}{2}\|x(t)-z\|^{2}+\lambda(t)\langle\dot{x}(t), x(t)-z\rangle+\frac{d}{d t} g_{z}(t) \leq \beta(t)\left(\Psi^{*}\left(\frac{p}{\beta(t)}\right)-\sigma_{\operatorname{argmin}} \Psi\left(\frac{p}{\beta(t)}\right)\right)$,

hence

$$
\begin{aligned}
\frac{d}{d t} g_{z} & \leq \beta(t)\left(\Psi^{*}\left(\frac{p}{\beta(t)}\right)-\sigma_{\operatorname{argmin}} \Psi\left(\frac{p}{\beta(t)}\right)\right)-\frac{\dot{\lambda}(t)}{2}\|x(t)-z\|^{2}+\lambda(t)\|\dot{x}(t)\| \cdot\|x(t)-z\| \\
& =G_{1}(t)+G_{2}(t),
\end{aligned}
$$

where

$$
G_{1}(t)=\beta(t)\left(\Psi^{*}\left(\frac{p}{\beta(t)}\right)-\sigma_{\operatorname{argmin}} \Psi\left(\frac{p}{\beta(t)}\right)\right)-\frac{\dot{\lambda}(t)}{2}\|x(t)-z\|^{2}
$$

and

$$
G_{2}(t)=\lambda(t)\|\dot{x}(t)\| \cdot\|x(t)-z\| .
$$

Now using that $x$ is bounded, from (ii) and $(H)$ we derive that

$$
G_{1} \in L^{1}([0,+\infty))
$$

and

$$
G_{2} \in L^{2}([0,+\infty)) .
$$

From (56), a direct application of Lemma 3 and Lemma 11(viii) yields

$$
\lim _{t \rightarrow+\infty} g_{z}(t)=0 .
$$

By combining this with Lemma 11(i) and the fact that $\lim _{t \rightarrow+\infty} \lambda(t)>0$, we conclude that there exists $\lim _{t \rightarrow+\infty}\|x(t)-z\| \in \mathbb{R}$. Since $z \in S$ has been chose arbitrary, the first condition of the Opial Lemma is fulfilled.

Let $\left(t_{n}\right)_{n \in \mathbb{N}}$ be a sequence of positive numbers such that $\lim _{n \rightarrow+\infty} t_{n}=+\infty$ and $x\left(t_{n}\right)$ converges weakly to $x_{\infty}$ as $n \rightarrow+\infty$. By using the weak lower semicontinuity of $\Psi$ and Lemma 12(ii) we obtain

$$
0 \leq \Psi\left(x_{\infty}\right) \leq \liminf _{n \rightarrow+\infty} \Psi\left(x\left(t_{n}\right)\right)=0,
$$

hence $x_{\infty} \in \operatorname{argmin} \Psi$. Moreover, the weak lower semicontinuity of $\Phi+\Theta$ and (55) yield

$$
(\Phi+\Theta)\left(x_{\infty}\right) \leq \liminf _{n \rightarrow+\infty}(\Phi+\Theta)\left(x\left(t_{n}\right)\right)=(\Phi+\Theta)(z),
$$

thus $x_{\infty} \in S$.

Remark 16 An anonymous reviewer raised the question whether the trajectories generated by (14) diverge in norm in case the optimization problem (13) has no solution. In the following we give a positive answer to this question. 
We assume that $\operatorname{dom} \Phi \cap \operatorname{argmin} \Psi \neq \varnothing$ (otherwise the optimization problem (13) is degenerate). We suppose that all the hypotheses of Theorem 15, excepting the assumption that the set of optimal solutions $S$ is nonemtpy, hold.

Let $(x, v):[0,+\infty) \rightarrow \mathcal{H} \times \mathcal{H}$ be a strong stable solution of (14) and assume that $x$ is bounded. In other words, there exists $M>0$ such that

$$
\|x(t)\| \leq M \forall t \geq 0 .
$$

Take $z \in \operatorname{dom} \Phi \cap \operatorname{argmin} \Psi$ and $r>0$ such that

$$
r>\max \{\|z\|, M\} .
$$

In the following we denote by $\bar{B}(0, r)$ the closed ball centered at origin with radius $r$. We will use as follows several times the fact that the normal cone to a set at an element belonging to the interior of this set reduces to $\{0\}$. Due to (58), we consequently have

$$
\partial\left(\Phi+\delta_{\bar{B}(0, r)}\right)(x(t))=\partial \Phi(x(t))+N_{\bar{B}(0, r)}(x(t))=\partial \Phi(x(t)) \forall t \geq 0 .
$$

This means that $(x, v)$ is a strong global solution of the system

$$
\left\{\begin{array}{l}
v(t) \in \partial\left(\Phi+\delta_{\bar{B}(0, r)}\right)(x(t)) \\
\lambda(t) \dot{x}(t)+\dot{v}(t)+v(t)+\nabla \Theta(x(t))+\beta(t) \nabla \Psi(x(t))=0
\end{array}\right.
$$

too.

This continuous system can be associated to the optimization problem

$$
\inf _{x \in \operatorname{argmin} \Psi}\left\{\Phi(x)+\delta_{\bar{B}(0, r)}(x)+\Theta(x)\right\} .
$$

Notice that the set of optimal solutions to (60) is nonempty, since its objective function is coercive. Further, by combining $(\widetilde{H})$ with $(58)$, we obtain:

$$
\partial\left(\Phi+\delta_{\bar{B}(0, r)}+\Theta+\delta_{\operatorname{argmin}} \Psi\right)=\partial\left(\Phi+\delta_{\bar{B}(0, r)}\right)+\nabla \Theta+N_{\operatorname{argmin}} \Psi .
$$

By applying Theorem 15 to the optimization problem (60) and the continuous system (59), it follows that there exists an optimal splution $\bar{u}$ to (60) such that $x(t)$ converges weakly to $\bar{u}$ as $t \rightarrow+\infty$. Due to the weak-lower semicontinuity of the norm function, from (57) and (58) it follows that $\bar{u}$ belongs to the interior of the closed ball $\bar{B}(0, r)$. Thus, from (61), (58) and $(\widetilde{H})$ we derive

$$
0 \in \partial\left(\Phi+\delta_{\bar{B}(0, r)}+\Theta+\delta_{\operatorname{argmin} \Psi}\right)(\bar{u})=\partial\left(\Phi+\Theta+\delta_{\operatorname{argmin}} \Psi\right)(\bar{u}) .
$$

The later implies that the set of optimal solutions to (13) is nonempty, which contradicts the assumption we made. In this way, our claim is proved.

In the last result we show that if the objective function of (13) is strongly convex, then the trajectory $x(\cdot)$ generated by (14) converges strongly to the unique optimal solution of (13).

Theorem 17 Assume that $\left(H_{\Psi}\right),\left(H_{\Theta}\right),\left(H_{\Phi}\right),\left(H_{\lambda}^{3}\right),\left(H_{\beta}^{3}\right),(H)$ and $(\tilde{H})$ hold and let $(x, v):[0,+\infty) \rightarrow \mathcal{H} \times \mathcal{H}$ be a strong stable solution of the dynamical system (14). If $\Phi+\Theta$ is strongly convex, then $x(t)$ converges strongly to the unique optimal solution of (13) as $t \rightarrow+\infty$.

Proof Let $\gamma>0$ be such that $\Phi+\Theta$ is $\gamma$-strongly convex. It is a well-known fact that in case the optimization problem (13) has a unique optimal solution, which we denote by $z$. 
From $(\tilde{H})$ there exists $p \in N_{\operatorname{argmin}} \Psi(z)$ such that $-p-\nabla \Theta(z) \in \partial \Phi(z)$. Consider again the functions $g_{z}, h_{z}$ defined in Lemma 11 .

By combining (41) with the stronger inequality

$$
(\Phi+\Theta)(x(t))-(\Phi+\Theta)(z) \geq\langle-p, x(t)-z\rangle+\frac{\gamma}{2}\|x(t)-z\|^{2} \forall t \in[0,+\infty),
$$

we obtain this time (see the proof of Lemma 11) for almost every $t \in[0,+\infty$ )

$$
\begin{aligned}
& \frac{d}{d t}\left(\frac{\lambda(t)}{2}\|x(t)-z\|^{2}+g_{z}(t)\right)+\frac{\gamma}{2}\|x(t)-z\|^{2}+\beta(t)\left(-\Psi^{*}\left(\frac{p}{\beta(t)}\right)+\sigma_{\operatorname{argmin}} \Psi\left(\frac{p}{\beta(t)}\right)\right) \\
\leq & \frac{d}{d t}\left(\frac{\lambda(t)}{2}\|x(t)-z\|^{2}+g_{z}(t)\right)+\frac{\gamma}{2}\|x(t)-z\|^{2}+\beta(t) \Psi(x(t))+\langle-p, x(t)-z\rangle \\
\leq & \frac{d}{d t}\left(\frac{\lambda(t)}{2}\|x(t)-z\|^{2}+g_{z}(t)\right)+(\Phi+\Theta)(x(t))-(\Phi+\Theta)(z)+\beta(t) \Psi(x(t)) \\
\leq & 0 .
\end{aligned}
$$

Taking into account $(H)$, by integration of the above inequality we obtain

$$
\int_{0}^{+\infty}\|x(t)-z\|^{2} d t<+\infty .
$$

Since according to the proof of Theorem $15, \lim _{t \rightarrow+\infty}\|x(t)-z\|$ exists, we conclude that $\|x(t)-z\|$ converges to 0 as $t \rightarrow+\infty$ and the proof is complete.

Remark 18 The results presented in this paper remain true even if the assumed growth condition is satisfied starting with a $t_{0} \geq 0$, that is, if there exists $t_{0} \geq 0$ such that

$$
0 \leq \dot{\beta}(t) \leq k \beta(t) \text { for almost every } t \in\left[t_{0},+\infty\right) .
$$

Acknowledgments Open access funding provided by Austrian Science Fund (FWF). The authors are thankful to two anonymous reviewers for comments and remarks which improved the presentation of the paper.

Open Access This article is distributed under the terms of the Creative Commons Attribution 4.0 International License (http://creativecommons.org/licenses/by/4.0/), which permits unrestricted use, distribution, and reproduction in any medium, provided you give appropriate credit to the original author(s) and the source, provide a link to the Creative Commons license, and indicate if changes were made.

\section{References}

1. Abbas, B.: An asymptotic viscosity selection result for the regularized Newton dynamic. arXiv: $1504.07793 \mathrm{v} 1$ (2015)

2. Abbas, B., Attouch, H., Svaiter, B.F.: Newton-like dynamics and forward-backward methods for structured monotone inclusions in Hilbert spaces. J. Optim. Theory Appl. 161(2), 331-360 (2014)

3. Attouch, H., Cabot, A., Czarnecki, M.-O.: Asymptotic behavior of nonautonomous monotone and subgradient evolution equations, to appear in Transactions of the American Mathematical Society, arXiv: 1601.00767 (2016)

4. Attouch, H., Czarnecki, M.-O.: Asymptotic control and stabilization of nonlinear oscillators with nonisolated equilibria. J. Differ. Equ. 179(1), 278-310 (2002)

5. Attouch, H., Czarnecki, M.-O.: Asymptotic behavior of coupled dynamical systems with multiscale aspects. J. Differ. Equ. 248(6), 1315-1344 (2010)

6. Attouch, H., Czarnecki, M.-O.: Asymptotic behavior of gradient-like dynamical systems involving inertia and multiscale aspects. J. Differ. Equ. 262(3), 2745-2770 (2017) 
7. Attouch, H., Czarnecki, M.-O., Peypouquet, J.: Prox-penalization and splitting methods for constrained variational problems. SIAM J. Optim. 21(1), 149-173 (2011)

8. Attouch, H., Czarnecki, M.-O., Peypouquet, J.: Coupling forward-backward with penalty schemes and parallel splitting for constrained variational inequalities. SIAM J. Optim. 21(4), 1251-1274 (2011)

9. Attouch, H., Maingé, P.-E.: Asymptotic behavior of second-order dissipative evolution equations combining potential with non-potential effects, ESAIM. Control, Optimisation and Calculus of Variations 17(3), 836-857 (2011)

10. Attouch, H., Svaiter, B.F.: A continuous dynamical Newton-like approach to solving monotone inclusions. SIAM J. Control. Optim. 49(2), 574-598 (2011)

11. Bauschke, H.H., Combettes, P.L.: Convex Analysis and Monotone Operator Theory in Hilbert Spaces CMS Books in Mathematics. Springer, New York (2011)

12. Banert, S., Bot, R.I.: Backward penalty schemes for monotone inclusion problems. J. Optim. Theory Appl. 166(3), 930-948 (2015)

13. Boț, R.I.: Conjugate duality in convex optimization. Lecture Notes in Economics and Mathematical Systems, vol. 637. Springer, Berlin Heidelberg (2010)

14. Boţ, R.I., Csetnek, E.R.: Second order dynamical systems associated to variational inequalities. Appl. Anal. 96(5), 799-809 (2017)

15. Bot, R.I., Csetnek, E.R.: Approaching the solving of constrained variational inequalities via penalty term-based dynamical systems. J. Math. Anal. Appl. 435(2), 1688-1700 (2016)

16. Boţ, R.I., Csetnek, E.R.: Forward-backward and Tseng's type penalty schemes for monotone inclusion problems. Set-Valued and Variational Analysis 22, 313-331 (2014)

17. Bot, R.I., Csetnek, E.R.: A Tseng's type penalty scheme for solving inclusion problems involving linearly composed and parallel-sum type monotone operators. Vietnam J. Math. 42(4), 451-465 (2014)

18. Brézis, H.: Opérateurs maximaux monotones et semi-groupes de contractions dans les espaces de Hilbert, North-Holland Mathematics Studies No. 5 Notas De Matemática, vol. 50. NorthHolland/Elsevier, New York (1973)

19. Haraux, A.: Systèmes Dynamiques Dissipatifs Et Applications, Recherches en Mathé- matiques Appliquéées, vol. 17. Masson, Paris (1991)

20. Noun, N., Peypouquet, J.: Forward-backward penalty scheme for constrained convex minimization without inf-compactness. J. Optim. Theory Appl. 158(3), 787-795 (2013)

21. Peypouquet, J.: Coupling the gradient method with a general exterior penalization scheme for convex minimization. J. Optim. Theory Appl. 153(1), 123-138 (2012)

22. Sontag, E.D.: Mathematical control theory systems. Deterministic Finite-Dimensional, Texts in Applied Mathematics, 2nd edn., vol. 6. Springer-Verlag, New York (1998)

23. Zalinescu, C.: Convex Analysis in General Vector Spaces. World Scientific, Singapore (2002) 